\title{
Influencing factors of aortic valve disease progression after mitral valve surgery
}

\author{
Zhengyang Sun ${ }^{1}$, Yanan Liang ${ }^{1}$, Shaobin $\mathrm{Li}^{2}$, Hui $\mathrm{Li}^{2}$, Yaoye Yuan ${ }^{1}$, Xiaoming Zou ${ }^{3}$, and \\ Yusheng Yan $^{2}$ \\ ${ }^{1}$ Southern Medical University \\ ${ }^{2}$ Zhujiang Hospital \\ ${ }^{3}$ The Fifth Affiliated Hospital, Southern Medical University
}

September 11, 2020

\begin{abstract}
Background:Some of the patients who have undergone mitral valve surgery may develop into aortic valve disease as time goes on. This study aimed to explore the influencing factors of the long-term outcome of the aortic valve after mitral valve surgery. Methods:Patients who underwent mitral valve surgery from 2010 to 2016 in Zhujiang Hospital were divided into two groups according to the outcome of the aortic valve. Those who had significant aortic valve disease progression after mitral valve surgery were classified into the progression group, and the other patients were classified into the non-progression group. Collected clinical data including echocardiography for statistical analysis. Results:Finally, 144 patients (59 males, 40.97\%) were enrolled, with an average age of $50.35 \pm 11.32$ years, and a median follow-up period of 4.2 years. Five related factors were determined by multivariate analysis, including tricuspid ring annuloplasty $(\mathrm{p}=0.010, \mathrm{OR}=0.12)$, left ventricular diameter $(\mathrm{p}=0.023, \mathrm{OR}=0.93)$, mild to moderate aortic valve disease $(\mathrm{p}=0.009, \mathrm{OR}=7.17)$, mitral prosthesis size $(\mathrm{p}=0.032,=\mathrm{OR}=2.51)$, aortic valve peak velocity $(\mathrm{p}=0.008, \mathrm{OR}=3.99)$. Conclusions: The progression of aortic valve disease after mitral valve surgery is related to a variety of factors. It may be mainly due to the increase of left ventricular preload and a series of hemodynamic changes after mitral valve surgery. For such patients, a comprehensive consideration is needed to formulate the surgical plan, rather than using the valve area as the sole criterion for determining whether to undergo aortic valve surgery at the same time or not.
\end{abstract}

\section{Introduction}

Mitral valve disease (MVD) is a common heart disease caused by rheumatic fever in most developing countries. For such diseases with clear indications, the most effective treatment is mitral valve surgery (repair, commissural incision, or valve replacement) [1]. However, the aortic valve disease (AVD) is present in up to $40 \%$ of patients with rheumatic MVD [2], and some patients who have undergone mitral valve surgery will develop AVD over time and may even need reoperation. Preoperative AVD in these patients is often mild to moderate. Concerning these patients, it has been controversial whether it is necessary to perform aortic valve surgery while dealing with the mitral valve. Since double valve replacement is more often associated with higher risk and poorer long-term survival than either of the two valves alone [3-4]. The decision of a surgical plan is often made by the surgeon during the operation. At present, there are few reports on the long-term outcomes and influencing factors of the aortic valve after single mitral valve surgery [5-8]. In this study, 144 patients who underwent mitral valve surgery (except for simultaneous aortic valve surgery) in Zhujiang Hospital of Southern Medical University from January 2010 to December 2016 were analyzed retrospectively. This study aimed to explore the predictors and to formulate clinical diagnosis and treatment strategies for the long-term progression of AVD after mitral valve surgery. 


\section{Materials and Methods}

\section{Study Population}

From January 1, 2010 to December 31, 2016, 241 patients received mitral valve repair/replacement(except for simultaneous aortic valve surgery)in Zhujiang Hospital of Southern Medical University. We classified all valvular lesions into mild, moderate and severe strictly according to the guidelines. Since the purpose of this study was to investigate the long-term effects of mitral valve surgery on the aortic valve, we also excluded patients who died within 30 days of surgery $(n=6)$, patients with significant progression of AVD within 30 days after surgery $(n=7$, The progression of these patients may be due to the direct factors of surgery) and patients who were followed for less than 2 years $(n=84)$. Finally, 144 eligible patients were selected for the study. A flow diagram of included and excluded patients is provided in the Figure 1. Demographic, clinical, and perioperative data of the patients were collected. Patients were examined by transthoracic echocardiography before surgery, within 2 weeks after surgery, 1 month, 2 months, and 6 months and then yearly for at least 2 years after discharge (the average follow-up time was $1554.72 \pm 759.03$ days). According to the results of follow-up echocardiography of the aortic valve, patients were divided into a progression group and a non-progression group. Those who meet the following conditions were defined as the progression group $(\mathrm{n}=25,17.36 \%): 1$. The follow-up of no or mild AVD progressed to moderate or severe aortic valve disease; 2 . The follow-up of moderate AVD progressed to severe. The non-progression group(n=119, $82.64 \%)$ was defined as: until the last follow-up echocardiography, there was no newly identified moderate AVD or no progression of AVD which was originally mild to moderate. The data collection ended in December 2019.

\section{Statistical Analysis}

All statistical analyses were performed using SPSS software (version 23.0, SPSS Inc., Chicago, Illinois). Continuous variables were expressed as mean $\pm \mathrm{SD}$ and compared using an unpaired Student t-test or the nonparametric Mann-Whitney test. Categorical variables were presented as counts and percentages and compared using chi-square statistics or the Fisher exact test. Binary logistic regression analysis was performed to identify the independent determinants of AVD progression. Variables for inclusion were carefully chosen to ensure the parsimony of the final models. Candidate variables that were considered clinically relevant (based on clinical experience and previous literature) or showed a univariate relationship with outcom $(\mathrm{p}<0.20)$ were included in the multivariable model. $\mathrm{P}$ value $<0.05$ was considered statistically significant.

\section{Results}

\section{Baseline Characteristics and Operative Data}

The baseline characteristics of the 25 patients in the progression group and the 119 patients in the nonprogression group were compared in Table 1. The mean age of the 144 patients included in the study was $50.35 \pm 11.32$ years, and most of these patients were female $(n=85,59.03 \%)$. The median follow-up time was 4.2 years. There were 13 patients with hypertension (9.0\%), 14 patients with cerebrovascular disease (9.7\%), 5 patients with diabetes $(3.5 \%)$, and 3 patients with renal insufficiency $(2.1 \%)$.

About surgery(Table 2), the mechanical valve was used in 129 cases, the biological valve in 11 cases, and ring plasty in 4 cases. 71 patients received tricuspid valve surgery (49.3\%), 6 patients received CABG (4.2\%), and 4 patients received atrial defect repair $(2.8 \%)$.

\section{Echocardiographic Findings}

Table 3 shows the echocardiographic data before the operation. 14 patients manifested as mitral stenosis (MS) (9.7\%), 64 patients manifested as mitral regurgitation (MR) (44.4\%), and the remaining 76 patients manifested as MR+MS (52.8\%). A total of 89 patients had mild to moderate AVD (61.8\%). In the progression group, 20 patients had mild AVD (80\%), 2 patients had moderate AVD (8\%, For these patients, due to the mild symptoms of AV before operation, we did not choose to undergo aortic valve surgery at the same time. ), and the remaining 3 patients had no AVD (12\%). 
As shown in Table4, finally 24 cases were simple aortic regurgitation (AR) (96\%) and 1 case was aortic stenosis (AS) + AR (4\%). Compared with the left atrial diameter before and after the operation, it was observed that in the non-progression group, the average left atrial diameter decreased from 47.72 to 43.75 $(\mathrm{p}<0.05)$, while in the progression group, the left atrial diameter decreased from 50.37 to $42.44(\mathrm{p}<0.05)$.

\section{Statistical Analysis}

The measurement data conforming to normal distribution and homogeneity of variance were tested by ttest, and the results showed that left ventricular diameter (LVD) $(45.83+-8.8 \mathrm{~mm})$ of the progression group was lower than that of the non-progression group $(48.61+-8.60 \mathrm{~mm}), \mathrm{p}=0.145<0.20$. The mitral valve peak velocity in the progression group $(2.07+-0.62 \mathrm{~m} / \mathrm{s})$ was higher than that in the non-progression group (1.83+$0.58 \mathrm{~m} / \mathrm{s}), \mathrm{p}=0.071<0.20$. The aortic valve velocity was tested by the Mann-Whitney U test. The results showed that the average rank of the progression group was 93.82, the non-progression group was 68.02, $\mathrm{U}=954.50, \mathrm{p}=0.005<0.20$. Although compared the patients in the progression group with the non-progression group, Hypertension ( 1 case, $4 \%$ vs 12 cases, $10.1 \%, \mathrm{p}=0.467>0.20$ ), atrial fibrillation (17 cases, $68 \%$ vs 73 cases, $61.3 \%, \mathrm{p}=0651>0.20), \operatorname{LVEF}(55.95+-4.38$ vs $57.49+-6.83, \mathrm{p}=0.323>0.20)$, None of the above results were significant. Referring to the results of Mentias S, Matsuyama K et al [9-12], these possible clinical related variables were also included in multivariate regression analysis. The final results are shown in Table 5 .

The results of the multivariable model have statistical significance, $\mathrm{x} 2=34.637, \mathrm{p}<0.001$. Among the 9 variables, tricuspid ring annuloplasty, LVD, mitral prosthesis size, the presence of mild to moderate AVD before the operation, and aortic valve velocity were statistically significant $(\mathrm{p}<0.05)$. Compared with untreated, patients who received tricuspid ring annuloplasty had a $90 \%$ lower risk of progression events. In order to facilitate multivariable analysis, we classified LVD into three grades according to clinical reference values $(<35 \mathrm{~mm}, 35-50 \mathrm{~mm},>50 \mathrm{~mm})$. With each increase of one grade, the risk is reduced by $73 \%$. Similarly, The aortic valve velocity was divided into three grades $(<1.0 \mathrm{~m} / \mathrm{kg}, 1.0-1.7 \mathrm{~m} / \mathrm{s},>1.7 \mathrm{~m} / \mathrm{s})$. With each increase of one grade, the risk increased by 3.70 times. The risk increased by 2.40 times for each additional level of mitral prosthesis size ( $25 \# 27 \# 29 \#)$. The risk of patients who had mild to moderate AVD before the operation was 7.28 times higher than those without.

\section{Discussion}

Of the 144 patients who underwent mitral valve surgery in this center, 25 patients progressed to significant AVD, which was similar to the results obtained by Kim DJ [8] in a retrospective analysis of 1231 patients $(13.2 \%)$.

The filling of the left ventricle in patients with MVD is often abnormal, masking the actual hemodynamic effect of the left ventricle, which is easy to misestimate the severity of AVD and actual peak velocity of the aortic valve by transthoracic echocardiography. Zitink RS [13] pointed out that for patients with severe MS complicated with mild to moderate AVD, the only certain method of quantitative evaluation to determine the degree of AVD is left heart catheterization, with simultaneous measurement of transvalvular pressure differences and blood flow. Besides, the presence of AVD before surgery means that the valve and its adjacent structures have shown pathological changes, so these patients are more likely to have further progress than patients without aortic valve involvement. As well as suggested by Choudhary SK [5] and Kim DJ [8] et al, the presence of mild to moderate AVD before the operation was a powerful risk factor for the progression of AVD.

Compared with aortic valve replacement, the hemodynamic changes of mitral valve replacement were more complex. Once the MVD is corrected, the blood flow from the left atrium to the left ventricle may be significantly increased(Especially for MS), leaving the left ventricle in a higher preload than before. It also changes the flow characteristics near the aortic valve as a result of changes in blood jet direction from the prosthetic mitral valve $[6,14]$. This means that the mechanical environment of the aortic valve will be changed. The mechanical environment of the aortic valve is complex, including shear stress, pressure, stretch force, and bending force. Some animal experiments and clinical observations have confirmed that there is 
a correlation between mechanical stress and heart valve biology. These mechanical changes cause a large number of biological responses, containing gene expression, protein activation, and phenotypic characteristics changes of valvular interstitial cells (VICs), thus regulating the tissue structure of the aortic valve [15-17]. For example, the elevated pressure of the aortic valve can lead to cellular dysfunction and extracellular matrix remodeling [18]. Specifically, it can change the phenotypic characteristics of aortic VICs, including a proportional increase in collagen synthesis, downregulation of the $\alpha$-smooth muscle actin, vimentin, and calponin, further lead to pathological changes such as valve thickening and calcification [19-21]. Under physiological conditions, the aortic valve can be extended by about $10 \%$ during diastole, and the tissue extensibility of the valve decreases with age. The reason may be that the continuous formation of collagen fibril increases the diameter of some fibers and reduces the compliance of valvular tissue, so the greater tensile force will be needed to produce the same extension. Batten et al reported that stretch force could regulate collagen synthesis in VICs through phenotypic transformation [22]. VICs synthesize collagen to change the stiffness of the valve to adapt to the stretch force, and the stiffness of the valve plays an important role in regulating the calcification of the valve [23]. Other studies have found that elevated pressure can increase the expression of vascular cell adhesion molecule-1 to induce inflammation [24].

Moreover, we found that prosthesis size is correlated with the progression of AVD in this study. The disease has a certain regional correlation in our center. In the south of China, most of the patients undergoing MV surgery are thin and elderly women, and the Implantation of an oversized prosthesis valve may be prone to cause valve mismatch (patient-prosthesis mismatch, PPM), which is more likely to lead to the progress of AVD. However, the clinical significance of mitral valve PPM has still been controversial. Ozyalcin S, Lam B [25-26], et al believed that PPM was related to long-term survival and recommended the implantation of a larger prosthesis. Nevertheless, other studies showed that PPM did not affect long-term survival [27]. According to the results of multivariable analysis, the implantation of a smaller size mitral prosthesis is a protective factor only in terms of the effect on the progression of AVD, which may also be related to increased left ventricular preload after surgery. Bolman RM [28] also suggested the implantation of smaller prosthetic valves in elderly, thin patients. Hence, it would seem that the aortic valve area should not be used as the single criterion for determining who is a candidate for aortic valve operation [11].

Heller SJ believes that in patients with chronic MVD, part of the myocardium is in a state of disuse due to long-standing underfilling of the left ventricle, resulting in its atrophy. It is considered that this is an adaptive change of left ventricle to preload reduction, which may be related to the function of cardiomyocytes and left ventricular remodeling in patients [29]. Although this state of atrophy is often reversible, due to the destruction of the structure of the mitral valve and left ventricle caused by surgery, coupled with intraoperative ischemia and reperfusion injury, it takes a long time for the left ventricle to recover to withstand blood from the left atrium, which also confirms the relationship between the endpoint event and the left ventricular size in the conclusion of our study. From the above view, for patients with smaller LVD before the operation, the replacement of the larger size prosthesis means that the left ventricle, which was in a state of enduring atrophy, will bear relatively more blood flow from the left atrium after the operation. It will make the left ventricle in a state of high preload for a long period after the operation, which may be one of the reasons for promoting the progression of AVD. Mentias et al [8] discovered from their large matched cohort studies that ventricular size criteria for prognosis were related to outcome "paradoxically" because those with larger volumes were more likely to be sent to life-prolonging surgery than those with smaller volumes. Although the authors did not report more detailed data.

According to another result of this study, tricuspid ring annuloplasty should be performed simultaneously during mitral valve surgery. Although there is just mild to moderate tricuspid regurgitation (TR) before the operation, it may quickly lead to the progression of TR and AVD if not treated. The occurrence of TR is mainly due to dilation of the annulus and enlargement of the right ventricle, which is usually secondary to the left heart failure, an overload of the right ventricle volume and pressure, and enlargement of the heart chamber caused by myocardial or valvular lesions. However, the interdependence of the left and right ventricles is extremely important for the function of the heart. In addition to having a common interventricular septum, their muscle fibers are continuous and can play a role as a mechanical complex. 
Furthermore, the two ventricles share a mutual biochemical environment, improving the function of both ventricles by improving both systemic and local concentrations of neurohormones [30]. Antunes MJ believed that untreated or recurrent TR can cause the right ventricle to dilate and the interventricular septum to move toward the left ventricle during diastole, results in a rise in pulmonary venous pressure and left ventricular diastolic pressure. These interrelated ventricular cavity pressure and volume alterations are called "restriction-dilatation syndrome" [31]. Therefore, it may be argued that simultaneous implantation of a tricuspid artificial ring can delay the progress of AVD by improving the right ventricular function.

\section{Limitations}

This study has the following limitations: first of all, there is no etiological classification of AVD during the analysis (rheumatism, degeneration, congenital, ischemic, infection and trauma, etc). Although in our center, the vast majority of patients underwent mitral valve surgery because of rheumatic changes, it is difficult to determine the pathological type of secondary aortic valve disease. Previous studies have shown that pathological types have different effects on the progression of the disease. Bahler RC [32] thought that the progression of AVD was positively correlated with the degree of valve calcification, for example. Wagner S [33] also pointed out that, in degenerative-calcific AS, valve stiffness and calcification are the primary factors affecting the progression of valve disease, and observed that the progression of congenital and rheumatic AVD is slower than that of degenerative diseases.

Another limitation is that 84 patients have been excluded from the statistical process because they were followed up for less than 2 years, and the outcome (death or other causes) of these patients could not be determined. Baria K et al [7] found that it would take about 14.1 years to undergo a secondary operation for patients with severe AVD after mitral valve surgery. However, the follow-up time of this study was just $4.3 \pm 2.1$ years. Data that may contribute to the results have not been observed.

The above reasons may lead to some deviations in the research results. To obtain more accurate results, it is necessary to further expand the data content and research cohort for follow-up research.

\section{Conclusion}

It has become a common practice to perform aortic valve replacement in symptomatic patients with severe AVD identified by transthoracic echocardiography. The guideline [34] states that it is reasonable for patients with moderate AR or AS (aortic velocity $3.0-3.9 \mathrm{~m} / \mathrm{s}$ ) in stage B to perform aortic valve replacement in conjunction with other cardiac surgery (class IIA of recommendation, level $\mathrm{C}$ of evidence). However, considering that double-valve surgery will prolong the duration of cardiopulmonary bypass and increase the risk of complications, prophylactic aortic valve replacement may not be recommended for mild AVD. Vaturi M et al also pointed out that mild AVD during mitral valve surgery rarely progresses to moderate or severe, even after a longer follow-up period [6,35]. Combined with the results of this study, it is suggested that patients with mild to moderate AVD, high peak velocity of the aortic valve $(>1.7 \mathrm{~m} / \mathrm{s})$ or small size left ventricular $(\mathrm{LVD}<35 \mathrm{~mm})$ should be considered as potential candidates for rapid progression of AVD, which need cardiotonic and diuresis treatment to reduce the load for a long time after the operation and closely followed up. Although this option may increase morbidity and mortality in a very small number of patients, it avoids the risk of double valve surgery for most patients. At the same time, it is suggested to relax the indications for simultaneous tricuspid valve repair during mitral valve surgery. In contrast, the selection of valve size should be as accurate and conservative as possible according to the patient's condition to avoid valve mismatch. Furthermore, in order to prove the accuracy of the above results, it is necessary to expand the cohort for further study.

Statement of Ethics The study was approved by the Ethics Committee of the Zhujiang Hospital of Southern Medical University, with waiver of informed consent.

Conflict of Interest Statement

The authors have no conflicts of interest to disclose. 
Funding Sources

There were no sources of funding.

Author Contributions

All authors fulfil the ICMJE criteria for authorship. ZY.S., YS.Y., and H.L designed the study. ZY.S., YN.L., and YY.Y were responsible for acquisition and analysis of data and drafting the paper. SB.L., YS.Y., and H.L were responsible for interpretation of data. YS.Y., and XM.Z critically revised the paper. All authors have read and approved the final version of the paper and take responsibility for the work.

\section{References}

[1] Tsang W. Recent advances in understanding and managing mitral valve disease[J]. F1000Res, 2019, 8.

[2] Unger P, Rosenhek R, Dedobbeleer C, et al. Management of multiple valve disease[J]. Heart, 2011, 97(4): $272-277$.

[3] Alsoufi B, Rao V, Borger MA, et al. Short- and Long-Term Results of Triple Valve Surgery in the Modern Era[J]. The Annals of Thoracic Surgery, 2006, 81(6): 2172-2178.

[4] Mueller XM, Tevaearai HT, Ruchat P, et al. Perioperative morbidity and mortality in combined aortic and mitral valve surgery[J]. The Journal of heart valve disease, 1997, 6(4): 387.

[5] Choudhary SK, Talwar S, Juneja R, et al. Fate of mild aortic valve disease after mitral valve intervention[J]. The Journal of Thoracic and Cardiovascular Surgery, 2001, 122(3): 583-586.

[6] Vaturi M, Porter A, Adler Y, et al. The natural history of aortic valve disease after mitral valve surgery[J]. J Am Coll Cardiol , 1999, 33(7): 2003-2008.

[7] Baria K, Kothari J, Rathod D. Prognosis of aortic valve disease following mitral valve surgery[J]. Polish Journal of Cardio-Thoracic Surgery, 2019, 16(2): 65-68.

[8] Kim DJ, Joo H, Lee S, et al. Natural history of mild aortic valve disease untreated at the time of rheumatic mitral valve replacement[J]. Interactive Cardio Vascular and Thoracic Surgery, 2018, 27(6): 828-835.

[9] Mentias A, Feng K, Alashi A, et al. Long-Term Outcomes in Patients With Aortic Regurgitation and Preserved Left Ventricular Ejection Fraction[J].J Am Coll Cardiol , 2016, 68(20): 2144-2153.

[10] Matsuyama K, Matsumoto M, Sugita T, et al. Predictors of residual tricuspid regurgitation after mitral valve surgery[J]. Ann Thorac Surg, 2003, 75(6): 1826-1828.

[11] Borer JSM. Aortic Valve Surgery for Aortic Regurgitation[J]. JACC (Journal of the American College of Cardiology) , 2016, 68(20): 2154-2156.

[12] Kennedy KD, Nishimura RA, Holmes DJ, et al. Natural history of moderate aortic stenosis[J]. J Am Coll Cardiol , 1991, 17(2): 313-319.

[13] Zitink RS, Piemme TE, Messer RJ, et al. The masking of aortic stenosis by mitral stenosis[J]. Am Heart $J, 1965,6922-30$.

[14] Totaro P, Argano V. Patient-prosthesis mismatch after mitral valve replacement: Myth or reality?[J]. The Journal of Thoracic and Cardiovascular Surgery, 2007, 134(3): 697-701.

[15] Weber KT, Sun Y, Katwa LC, et al. Connective Tissue and Repair in the Heart[J]. Annals of the New York Academy of Sciences, 1995, 752(1): 286-299.

[16] Schneider PJ, Deck JD. Tissue and cell renewal in the natural aortic valve of rats: an autoradiographic study[J]. Cardiovascular research, 1981, 15(4): 181-189. 
[17] Willems I E, Havenith M G , Smits J F , et al. Structural alterations in heart valves during left ventricular pressure overload in the rat [J]. Laboratory Investigation A Journal of Technical Methods \& Pathology, 1994, 71(1): 127-33.

[18] Rajamannan NM, Bonow RO, Rahimtoola SH. Calcific aortic stenosis: an update[J]. Nature Clinical Practice Cardiovascular Medicine, 2007, 4(5): 254-262.

[19] Weston MW, Yoganathan AP. Biosynthetic Activity in Heart Valve Leaflets in Response to In Vitro Flow Environments[J]. Annals of Biomedical Engineering, 2001, 29(9): 752-763.

[20] Xing Y, Warnock JN, He Z, et al. Cyclic Pressure Affects the Biological Properties of Porcine Aortic Valve Leaflets in a Magnitude and Frequency Dependent Manner[J]. Annals of Biomedical Engineering, 2004, 32(11): 1461-1470.

[21] Thayer P, Balachandran K, Rathan S, et al. The Effects of Combined Cyclic Stretch and Pressure on the Aortic Valve Interstitial Cell Phenotype[J]. Annals of Biomedical Engineering, 2011, 39(6): 1654-1667.

[22] Batten P, Ku CH, Johnson PH, et al. Collagen synthesis by mesenchymal stem cells and aortic valve interstitial cells in response to mechanical stretch [J].Cardiovascular Research, 2006, 71(3): 548-556.

[23] Chen JH, Yip CYY, Zhao R, Simmons CA. Calcification by valve interstitial cells is regulated by the stiffness of the extracellular matrix[J]. Arteriosclerosis Thrombosis and Vascular Biology, 2009, 29(6): 936942.

[24] Warnock JN, Burgess SC, Shack A, et al. Differential immediate-early gene responses to elevated pressure in porcine aortic valve interstitial cells[J]. The Journal of heart valve disease, 2006, 15(1): 34.

[25] Ozyalcin S, Vural KM, Colak A. Mitral Patient-Prosthesis Mismatch Predicts Suboptimal Hemodynamic Recovery after Mitral Valve Replacement[J]. J Heart Valve Dis , 2016, 25(5): 589-595.

[26] Lam B, Chan V, Hendry P, et al. The impact of patient-prosthesis mismatch on late outcomes after mitral valve replacement[J]. The Journal of Thoracic and Cardiovascular Surgery, 2007, 133(6): 1464-1473.

[27] Capoulase R, Zeng X, Overbey JR, et al. Impact of Left Ventricular to Mitral Valve Ring Mismatch on Recurrent Ischemic Mitral Regurgitation After Ring Annuloplasty[J]. Circulation, 2016, 134(17): 1247-1256.

[28] Bolman RM. Survival After Mitral Valve Replacement[J]. Circulation , 2007, 115(11): 1336-1338.

[29] Heller SJ, Carleton RA. Abnormal Left Ventricular Contraction in Patients with Mitral Stenosis[J]. Circulation, 1970, 42(6): 1099-1110.

[30] Ho SY. Anatomy, echocardiography, and normal right ventricular dimensions[J]. Heart , 2006, 92(suppl_1): i2-i13.

[31] Antunes MJ, Barlow JB. Management of tricuspid valve regurgitation[J].Heart , 2005, 93(2): 271-276.

[32] Bahler RC, Desser DR, Finkelhor RS, et al. Factors leading to progression of valvular aortic stenosis[J]. Am J Cardiol , 1999, 84(9): 1044-1048.

[33] Wagner S, Selzer A. Patterns of progression of aortic stenosis: a longitudinal hemodynamic study[J]. Circulation , 1982, 65(4): 709-712.

[34] Nishimura RA, Otto CM, Bonow RO, et al. 2014 AHA/ACC Guideline for the Management of Patients With Valvular Heart Disease[J]. Circulation, 2014, 129(23).

[35] Ha JW, Choi SH, Chang BC, et al. Is prophylactic aortic valve replacement indicated during mitral valve surgery for mild to moderate aortic valve disease?[J]. Ann Thorac Surg, 2002, 74(4): 1115-1119. 


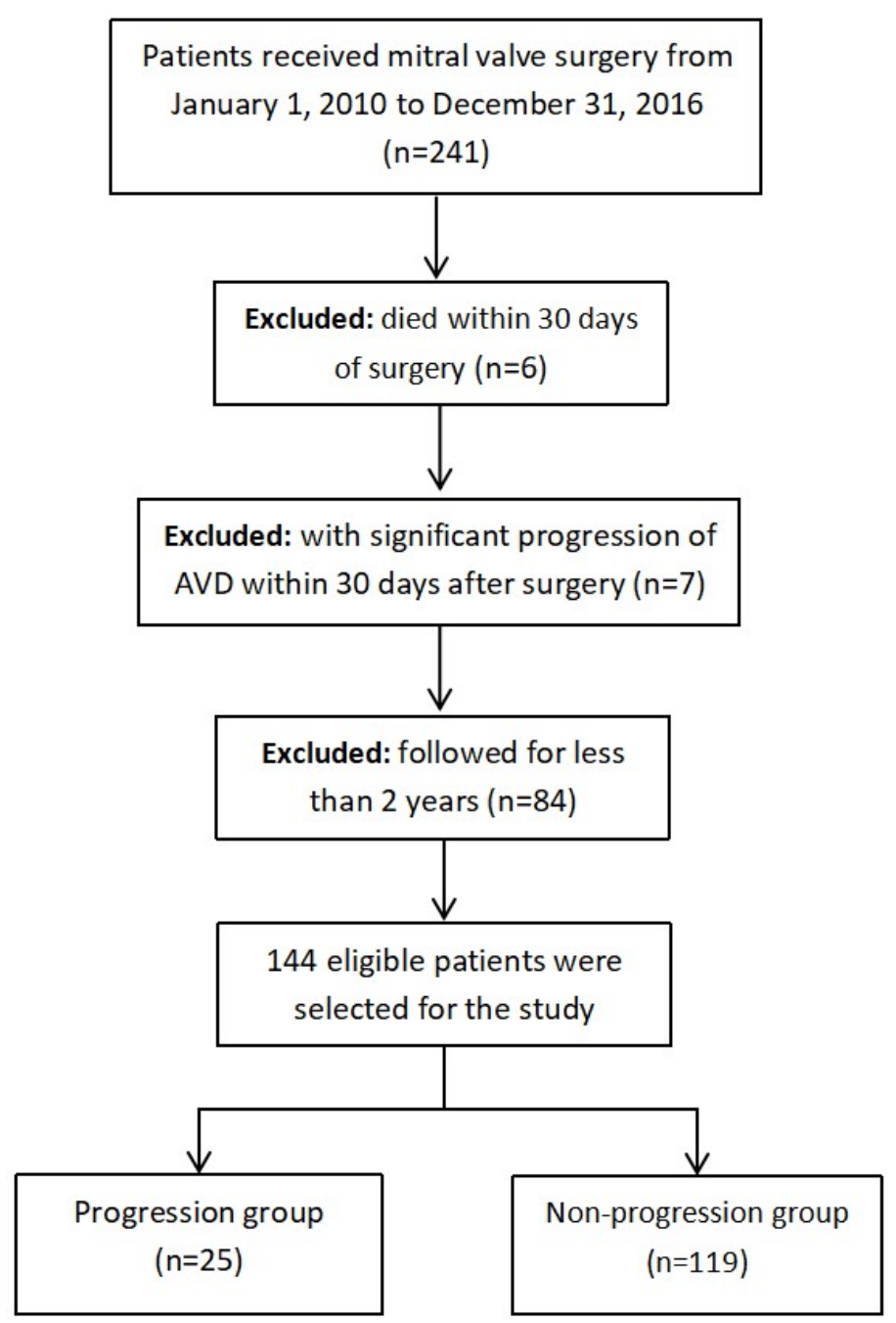

\section{Hosted file}

Table1.docx available at https://authorea.com/users/357564/articles/480064-influencingfactors-of-aortic-valve-disease-progression-after-mitral-valve-surgery

\section{Hosted file}

Table2.docx available at https://authorea.com/users/357564/articles/480064-influencingfactors-of-aortic-valve-disease-progression-after-mitral-valve-surgery

\section{Hosted file}

Table3.docx available at https://authorea.com/users/357564/articles/480064-influencingfactors-of-aortic-valve-disease-progression-after-mitral-valve-surgery

\section{Hosted file}


Table4.docx available at https://authorea.com/users/357564/articles/480064-influencingfactors-of-aortic-valve-disease-progression-after-mitral-valve-surgery

\section{Hosted file}

Table5.docx available at https://authorea.com/users/357564/articles/480064-influencingfactors-of-aortic-valve-disease-progression-after-mitral-valve-surgery 